\title{
Electron transport in semiconducting carbon nanotubes with hetero-metallic contacts
}

\author{
Yongqiang Xue ${ }^{1, *}$ and Mark A. Ratner ${ }^{2}$ \\ ${ }^{1}$ College of Nanoscale Science and Engineering, University at Albany, \\ State University of New York, Albany, New York 12203, USA \\ ${ }^{2}$ Department of Chemistry and Materials Research Center, \\ Northwestern University, Evanston, Illinois 60208, USA
}

(Dated: November 7, 2018)

\begin{abstract}
We present an atomistic self-consistent study of the electronic and transport properties of semiconducting carbon nanotube in contact with metal electrodes of different work functions, which shows simultaneous electron and hole doping inside the nanotube junction through contact-induced charge transfer. We find that the band lineup in the nanotube bulk region is determined by the effective work function difference between the nanotube channel and source/drain electrodes, while electron transmission through the SWNT junction is affected by the local band structure modulation at the two metal-nanotube interfaces, leading to an effective decoupling of interface and bulk effects in electron transport through nanotube junction devices.

PACS numbers: 73.63.-b,73.40.-c,85.65.+h
\end{abstract}

Devices based on single-wall carbon nanotubes (SWNTs) ${ }^{1.2}$ have been progressing in a fast pace, e.g., the performance of carbon nanotube field-effect transistors (NTFET) is approaching that of the state-of-theart silicon Metal-Oxide-Semiconductor field-effect transistors (MOSFET) 3.4.5 But a general consensus on the physical mechanisms and theoretical models remains to appear. A point of continuing controversy in NTFET has been the effect of Schottky barriers at the metal-SWNT interface ${ }^{6.7}$ Since SWNTs are atomic-scale nanostructures in both the axial and the circumferential dimensions, any barrier that may form at the interface has a finite thickness and a finite width $3,8,9$ In general a microscopic treatment of both the source/drain and gate field modulation effect will be needed to account for faithfully the atomistic nature of the electronic processes in NTFET.

Since the characteristics of the NTFETs depend sensitively on the gate geometry, ${ }^{3}$ a thorough understanding of the Schottky barrier effect in the simpler two-terminal metal-SWNT-metal junction devices is essential in elucidating the switching effect caused by applying a finite gate voltage. ${ }^{8}$ As a basic device building block, the twoterminal device is also of interests for applications in electromechanical and electrochemical sensors, where the conduction properties of the SWNT junctions are modulated by mechanical strain ${ }^{10}$ or molecular adsorption respectively 11 Previous works have considered symmetric SWNT junctions with different contact geometries ${ }^{8}$ Here we consider SWNT in contact with metallic electrodes of different work functions. Such hetero-metallic junctions are of interests since: (1) The electrode work function difference leads to a contact potential and finite electric field (built-in field) across the junction at equilibrium. A self-consistent analysis of the hetero-metallic junction can shed light on the screening of the applied field by the SWNT channel and the corresponding band bending effect even at zero bias; (2) For SWNTs not intentionally doped, electron and hole doping can be induced si- multaneously inside the channel by contacting with high and low work function metals; (3) Since the metallurgy of the metal-SWNT contact is different at the two interfaces, the asymmetric device structure may facilitate separating interface effect on electron transport from the intrinsic property of the SWNT channel.

The hetero-metallic SWNT junction is shown schematically in Fig. 1] where the ends of an infinitely long SWNT wire are buried inside two semi-infinite metallic electrodes with different work functions. The embedded contact scheme is favorable for the formation of low-resistance contact. For simplicity, we assume the embedded parts of the SWNT are surrounded entirely by the metals with overall cylindrical symmetry around the SWNT axis. ${ }^{12}$ For comparison with previous work on symmetric SWNT junctions, we investigate $(10,0)$ SWNTs (with work function of $4.5 \mathrm{eV}^{1}$ ) in contact with gold $(\mathrm{Au})$ and titanium (Ti) electrodes (with work functions of 5.1 and $4.33 \mathrm{eV}$ respectively for polycrystalline materials $\left.{ }^{13}\right)$. Choosing the electrostatic potential energy in the middle of the junction and far away from the cylindrical surface of the SWNT as the energy reference, the Fermi-level of the Au-SWNT-Ti junction is the negative of the average metal work functions $E_{F}=-4.715$ $\mathrm{eV}$. The SWNT channel length investigated ranges from $L=2.0,4.1,8.4,12.6,16.9 \mathrm{~nm}$ to $21.2 \mathrm{~nm}$, corresponding to number of unit cells of $5,10,20,30,40$ and 50 respectively. We calculate the transport characteristics within the coherent transport regime, as appropriate for such short nanotubes 14

Using a Green's function based self-consistent tightbinding (SCTB) theory, we analyze the Schottky barrier effect by examining the electrostatics, the band lineup and the transport characteristics of the heterometallic SWNT junction as a function of the SWNT channel length. The SCTB model is essentially the semi-empirical implementation of the self-consistent Matrix Green's function method for $a b$ initio modeling of molecular-scale devices, 15 which takes fully into account 
the three-dimensional electrostatics and the atomic-scale electronic structure of the SWNT junctions and has been described in detail elsewhere ${ }^{8.16}$ The SCTB model starts with the semi-empirical Hamiltonian $H_{0}$ of the bare $(10,0)$ SWNT wire using the Extended Huckel Theory (EHT) with non-orthogonal $(s p)$ basis sets $\phi_{m}(\vec{r}) \cdot 17$ We describe the interaction between the SWNT channel and the rest of the junction using matrix self-energy operators and calculate the density matrix $\rho_{i j}$ and therefore the electron density of the equilibrium SWNT junction from

$$
\begin{aligned}
G^{R} & =\left\{\left(E+i 0^{+}\right) S-H-\Sigma_{L}(E)-\Sigma_{L ; N T}(E)-\Sigma_{R}(E)-\right. \\
\rho & =\int \frac{d E}{2 \pi} \operatorname{Imag}\left[G^{R}\right](E) f\left(E-E_{F}\right) .
\end{aligned}
$$

Here $S$ is overlap matrix and $f\left(E-E_{F}\right)$ is the Fermi distribution in the electrodes. Compared to the symmetric SWNT junctions, here the Hamiltonian describing the SWNT channel $H=H_{0}+\delta V[\delta \rho]+V_{\text {ext }}$ includes the contact potential $V_{\text {ext }}$ (taken as linear voltage ramp here) in addition to the charge-transfer induced electrostatic potential change $\delta V(\delta \rho$ is the density of transferred charge).

The calculated charge transfer per atom and electrostatic potential change along the cylindrical surface of the SWNT for the Au-SWNT-Ti junction are shown in Fig. (2). Previous works 8 have shown that by contacting to the high (low)-work function metal Au (Ti), hole (electron) doping is induced inside the SWNT channel. Here we find simultaneous electron and hole doping inside the SWNT channel for the hetero-metallic AuSWNT-Ti junction (lower figure in Fig. 2(a)). Since the magnitude of hole doping inside the Au-SWNT-Au junction $\left(\approx-5.6 \times 10^{-4} /\right.$ atom $)$ is much larger than that of the electron doping inside the Ti-SWNT-Ti junction $\left(\approx 3 \times 10^{-5} /\right.$ atom $)$ due to the larger work function difference, the majority of the channel remains hole-doped inside the Au-SWNT-Ti junction. Due to the localized nature of interface bonding, the charge transfer pattern immediately adjacent to the $\mathrm{Au}(\mathrm{Ti})-\mathrm{SWNT}$ interface remains similar to that of the Au-SWNT-Au (Ti-SWNTTi) junction both in magnitude and shape. The shortwavelength oscillation in the transferred charge inside the SWNT channel reflects the atomic-scale variation of charge density within the unit cell of the SWNT 8,18

The contact-induced doping affects the transport characteristics by modulating the electrostatic potential profile along the SWNT junction. We find that inside the SWNT channel, the built-in electric field is screened effectively by the delocalized $\pi$-electron of carbon. So the net electrostatic potential change along the cylindrical surface $\left(V_{\text {ext }}+\delta V[\delta \rho]\right)$ is much more flat than the linear voltage ramp denoting the contact potential except close to the metal-SWNT interface (lower figure of Fig. 2(b)), where its shape remains qualitatively similar to that at the $\mathrm{Au}(\mathrm{Ti})-\mathrm{SWNT}$ interface of the $\mathrm{Au}$ SWNT-Au (Ti-SWNT-Ti) junction. Due to the confined cylindrical structure of the SWNT channel, the charge- transfer induced electrostatic potential change $\delta V$ decays rapidly in the direction perpendicular to the SWNT axis. This has led to a different physical picture of band bending in symmetric SWNT junctions ${ }^{8}$ In particular, the band lineup inside the SWNT channel has been found to depend mainly on the metal work function, while interaction across the metal-SWNT interface modulates the band structure close to the interface without affecting the band lineup scheme in the middle of the channel. Similar physical picture applies to the hetero-metallic SWNT junction, where we find that the band lineup in the middle of the Au-SWNT-Ti junction is essentially identical

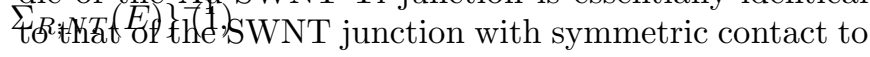
metals with (byork function of $4.715 \mathrm{eV}$. This is examined through the focal-density-of-states (LDOS) of the SWNT channel as a function of position along the SWNT axis in Figs. 3 and 4

The coupling across the metal-SWNT interface and the corresponding strong local field variation immediately adjacent to the Ti-SWNT interface has a strong effect on the SWNT band structure there, which extends to $\sim 4 \mathrm{~nm}$ away from the interface (Fig. 3(a)). The band structure modulation at the Au side is weaker. For the 40-unit cell $(16.9 \mathrm{~nm})$ SWNT, the band structure in the middle of the SWNT junction remains essentially unaffected. This is shown in Fig. 4 where we compare the LDOS of the Au-SWNT-Ti junction in the left end, the right end and the middle of the SWNT channel with the corresponding LDOS of the Au-SWNT-Au, Ti-SWNTTi junction and the bulk (infinitely long) SWNT wire respectively. Since the magnitude of the build-in electric field is smaller than the charge-transfer induced local field at the metal-SWNT interface, the LDOS at the two ends of the SWNT channel remain qualitatively similar to that of the symmetric SWNT junction (Figs. 4(a) and 4(c)). Note that the LDOS plotted here has been energetically shifted so that the SWNT bands in the middle of the hetero-metallic junction line up with those of the symmetric SWNT junctions.

The above separation of band lineup scheme at the interface and in the interior of the SWNT junction implies that in NTFETs, the gate segments controlling the device interiors affect the device operation through effective modulation of the work function difference between the source/drain electrode and the bulk portion of the SWNT channel (applying a finite gate voltage to the SWNT bulk leads to an effective modulation of its work function relative to the source/drain electrodes), while the gate segments at the metal-SWNT interfaces affect the device operation by controlling charge injection into the device interior through local modulation of the SWNT band structure and Schottky barrier shapes including height, width and thickness, in agreement with recent lateral scaling analysis of gate-modulation effect ${ }^{19}$ and interface chemical treatment effect in Schottky barrier NTFETs ${ }^{20}$ Note that since the band structure modulation at the metal-SWNT interface can extend up to $\sim 4 \mathrm{~nm}$ into the interior of the SWNT junction, it may be 
readily resolved using scanning nanoprobe techniques ${ }^{21}$

The Schottky barrier effect at the metal-SWNT interface can also be analyzed through the length-dependent conductance and current-voltage (I-V) characteristics of the Au-SWNT-Ti junction, which are calculated using the Landauer formula ${ }^{15} G=\frac{2 e^{2}}{h} \int d E T(E)\left[-\frac{d f}{d E}(E-\right.$ $\left.\left.E_{F}\right)\right]=G_{T u}+G_{T h}$ and $I=\int_{-\infty}^{+\infty} d E \frac{2 e}{h} T(E, V)[f(E-$ $\left.\left(E_{F}+e V / 2\right)\right)-\left[f\left(E-\left(E_{F}-e V / 2\right)\right)\right]=I_{T u}+I_{T h}$ and separated into tunneling and thermal-activation contributions as $G_{T u}=\frac{2 e^{2}}{h} T\left(E_{F}\right), G_{T h}=G-G_{T u}$ and $I_{T u}=\frac{2 e}{h} \int_{E_{F}-e V / 2}^{E_{F}+e V / 2} T(E, V) d E, I_{T h}=I-I_{T u}{ }^{8,22}$

In general the transmission function is voltagedependent due to the self-consistent screening of the source-drain field by the SWNT channel at each bias voltage. Since in the case of voltage dropping mostly across the interface, the transmission coefficient is approximately voltage-independent at low-bias, $\stackrel{22}{=}$ here we calculate the I-V characteristics using the equilibrium transmission coefficient instead of the full self-consistent calculation at each bias voltage. We find that the conductance of the Au-SWNT-Ti junction shows a transition from tunneling-dominated to thermal activationdominated regime with increasing channel length, but the length where this occurs is longer than those of the symmetric Au/Ti-SWNT-Au/Ti junctions (Fig. 囵(a)). This is partly due to the fact that the Fermi-level is closer to the mid-gap of the SWNT band inside the channel, partly due to the reduced transmission close to the valence-band edge (Fig. [4 (d)) caused by the band structure modulation at the Ti-SWNT interface. Due to the finite number of conduction channels, the increase of the conductance with temperature is rather slow (Fig. [5(b)) $\stackrel{8}{\underline{8}}$ The relative contribution of tunneling and thermal-activation to the room-temperature $\mathrm{I}-\mathrm{V}$ characteristics is shown in Figs. 5(c) and 5(d) for the 20- and 40-unit cell long (8.4 and $16.9 \mathrm{~nm}$ ) SWNT respectively, where we see that thermal-activation contribution increases rapidly with bias voltage for the 20-unit cell SWNT junction while the thermal-activation contribution dominates the I-V characteristics at all bias voltages for the 40-unit cell SWNT.

In conclusion, we have presented an atomistic realspace analysis of Schottky barrier effect in the twoterminal SWNT junction with hetero-metallic contacts, which shows an effective decoupling of interface and bulk effects. Further analysis is needed that treat both the gate and source/drain fields self-consistently in the real space to achieve a thorough understanding of NTFETs.
* Author to whom correspondence should be addressed. E-mail: yxue@uamail.albany.edu. URL: http://www.albany.edu/ yx152122

1 Dekker C 1999 Phys. Today 52(5) 22

2 Bachtold A, Haley P, Nakanishi T and Dekker C 2001 Science 2941317

3 Avouris Ph, Appenzellaer J, Martel R and Wind S J 2003 Proc. IEEE 911772

4 Javey A, Guo J, Wang Q, Lundstrom M and Dai H 2003 Nature 424 654; Javey A, Guo J, Paulsson M, Wang Q, Mann D, Lundstrom M and Dai H 2004 Phys. Rev. Lett. 92106804

5 Yaish Y, Park J-Y, Rosenblatt S, Sazonova V, Brink M and McEuen P L 2004 Phys. Rev. Lett. 9246401

6 Xue Y and Datta S 1999 Phys. Rev. Lett. 83 4844; Leónard F and Tersoff J 2000 Phys. Rev. Lett. 84 4693; Odintsov A A 2000 Phys. Rev. Lett. 85 150; Nakanishi T, Bachtold A and Dekker C 2002 Phys. Rev. B 6673307

7 Heinze S, Tersoff J, Martel R, Derycke V, Appenzeller J and Avouris Ph 2002 Phys. Rev. Lett. 89 106801; Appenzeller J, Knoch J, Radosavljević M and Avouris Ph 2004 ibid. 92226802

8 Xue Y and Ratner M A 2003 Appl. Phys. Lett. 83 2429; Xue Y and Ratner M A 2004 Phys. Rev. B 69 161402(R); Xue Y and Ratner M A 2004 Phys. Rev. B In Press (Preprint cond-mat/0405465)

9 Appenzeller J, Radosavljević M, Knoch J and Avouris Ph 2004 Phys. Rev. Lett. 9248301

10 Minot E D, Yaish Y, Sazonova V, Park J-Y, Brink M and
McEuen P L 2003 Phys. Rev. Lett. 90 156401; Cao J, Wang Q and Dai H 2003 Phys.Rev. Lett. 90157601

11 Chiu P-W, Kaempgen M, and Roth S 2004 Phys. Rev. Lett. 92 246802; Chen G, Bandow S, Margine E R, Nisoli C, Kolmogorov A N, Crespi V H, Gupta R, Sumanasekera G U, Iijima S and Eklund P C 2003 Phys. Rev. Lett. 90 257403

12 Experimentally low-resistance contacts can be obtained either by growing SWNT's directly out of the predefined catalyst islands and subsequently covering the catalyst islands with metallic contact pads (Ref. 4) or by using standard lithography and lift-off techniques with subsequent annealing at high-temperature (Ref. 7). In both cases, the ends of the long SWNT wires are surrounded entirely by the metals with strong metal-SWNT surface chemical bonding, although the exact atomic structure of the metal-SWNT interface remains unclear. Contacts can also be formed by depositing SWNT on top of the predefined metallic electrodes and side-contacted to the surfaces of the metals (side-contact scheme), which corresponds to the weak coupling limit due to the weak van der Waals bonding in the side-contact geometry leading to high contact resistance (Ref. 3). Other types of contact may also exist corresponding to intermediate coupling strength. The contact geometry chosen in this work thus serves as a simplified model of the low-resistance contact. A comprehensive study of the contact effects in SWNT junction devices is currently under way and will be reported in a future publication.

131994 CRC Handbook of Chemistry and Physics (CRC 
Press, Boca Raton)

14 Yao Z, Kane C L and Dekker C 2000 Phys. Rev. Lett. 84 2941; Park J-Y, Rosenblatt S, Yaish Y, Sazonova V, Ustunel H, Braig S, Arias T A, Brouwer P and McEuen P L 2004 Nano Lett. 4517

15 Xue Y, Datta S and Ratner M A 2002 Chem. Phys. 281 151; See also Datta S 1995 Electron Transport in Mesoscopic Systems (Cambridge University Press, Cambridge)

16 The SWNT-metal interaction arises from one discrete cylindrical shell of metal atoms, surrounded by the bulk metal and treated using the Green's function method as detailed in Ref. 15. We use a SWNT-metal surface distance of $2.0(\AA)$, close to the average inter-atomic spacing in the SWNTs and metals.

17 Hoffmann R 1988 Rev. Mod. Phys. 60 601; Rochefort A,
Salahub D R and Avouris Ph 1999 J. Phys. Chem. B 103 641

18 Leónard F and Tersoff J 2002 Appl. Phys. Lett. 814835

19 Wind S J, Appenzeller J, and Avouris Ph 2003 Phys. Rev. Lett. 9158301

20 Auvray S, Borghetti J, Goffman M F, Filoramo A, Derycke V, Bourgoin J P and Jost O 2004 Appl. Phys. Lett. 845106

21 Freitag M, Radosavljević M, Clauss W and Johnson A T 2000 Phys. Rev. B 62 R2307; Venema L C, Janssen J W, Buitelaar M R, Wild'oer J W G, Lemay S G, Kouwenhoven L P and Dekker C 2000 Phys. Rev. B 625238

22 Xue Y and Ratner M A 2003 Phys. Rev. B 68 115406; Xue Y and Ratner M A 2004 Phys. Rev. B 6985403 
(a)

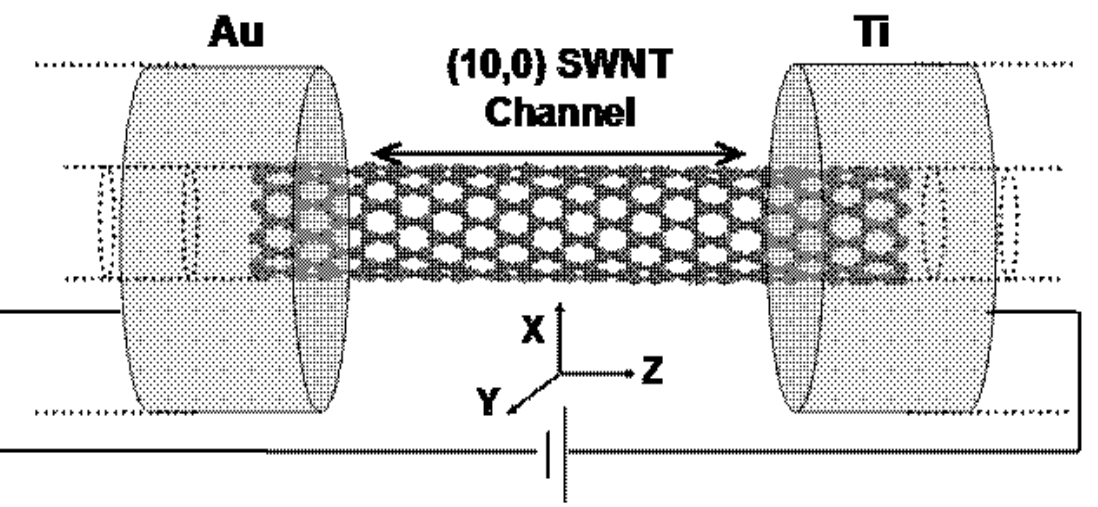

(b)

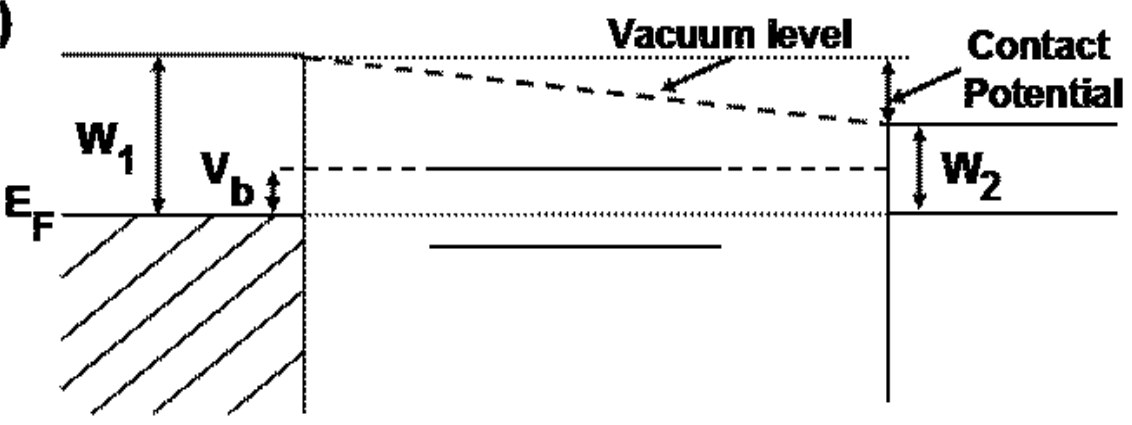

FIG. 1: (Color online) (a) Schematic illustration of the AuSWNT-Ti junction. The ends of the long SWNT wire are surrounded entirely by the semi-infinite electrodes, with only a finite segment being sandwiched between the electrodes (defined as the channel). Also shown is the coordinate system of the nanotube junction. (b) Schematic illustration of the band diagram in the Au-SWNT-Ti junction. The band alignment in the middle of the SWNT junction is determined by the average of the metal work functions. $W_{1(2)}, E_{F}$ denote the work functions and Fermi-level of the bi-metallic junction. 


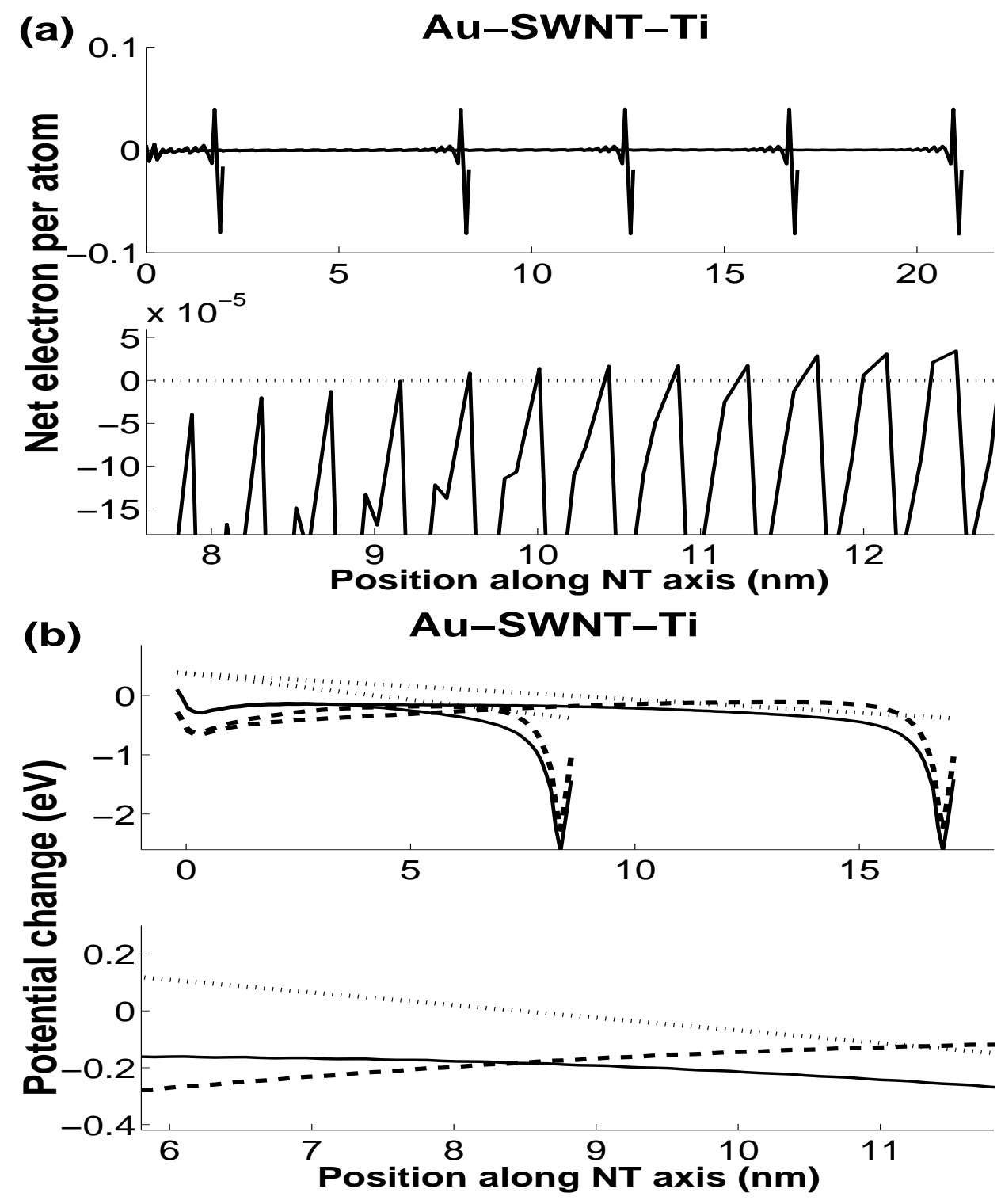

FIG. 2: Electrostatics of the Au-SWNT-Ti junction for SWNT channels of different lengths. (a) Upper figure shows transferred charge per atom as a function of position along the SWNT axis for SWNT channel lengths of 2.0, 8.412.6, 16.9 and $21.2 \mathrm{~nm}$. Lower figure shows the magnified view of the transferred charge in the middle of the channel for the longest (21.2 nm) SWNT studied. (b) Upper figure shows the electrostatic potential change at the cylindrical surface of the 20 $(8.4 \mathrm{~nm})$ and $40-$ unitcell $(16.9 \mathrm{~nm})$ SWNTs studied. The dotted line denote the linear voltage ramp $V_{\text {ext }}$ (contact potential) due to the work function difference of gold and titanium. The dashed line show the charge-transfer induced electrostatic potential change $\delta V(\delta \rho)$. The solid line shows the net electrostatic potential change $V_{e x t}+\delta V$. Lower figure shows the magnified view of the electrostatic potential change in the middle of the 40-unitcell SWNT junction. 


\section{(a) L=16.9 nm: Self-Consistent}

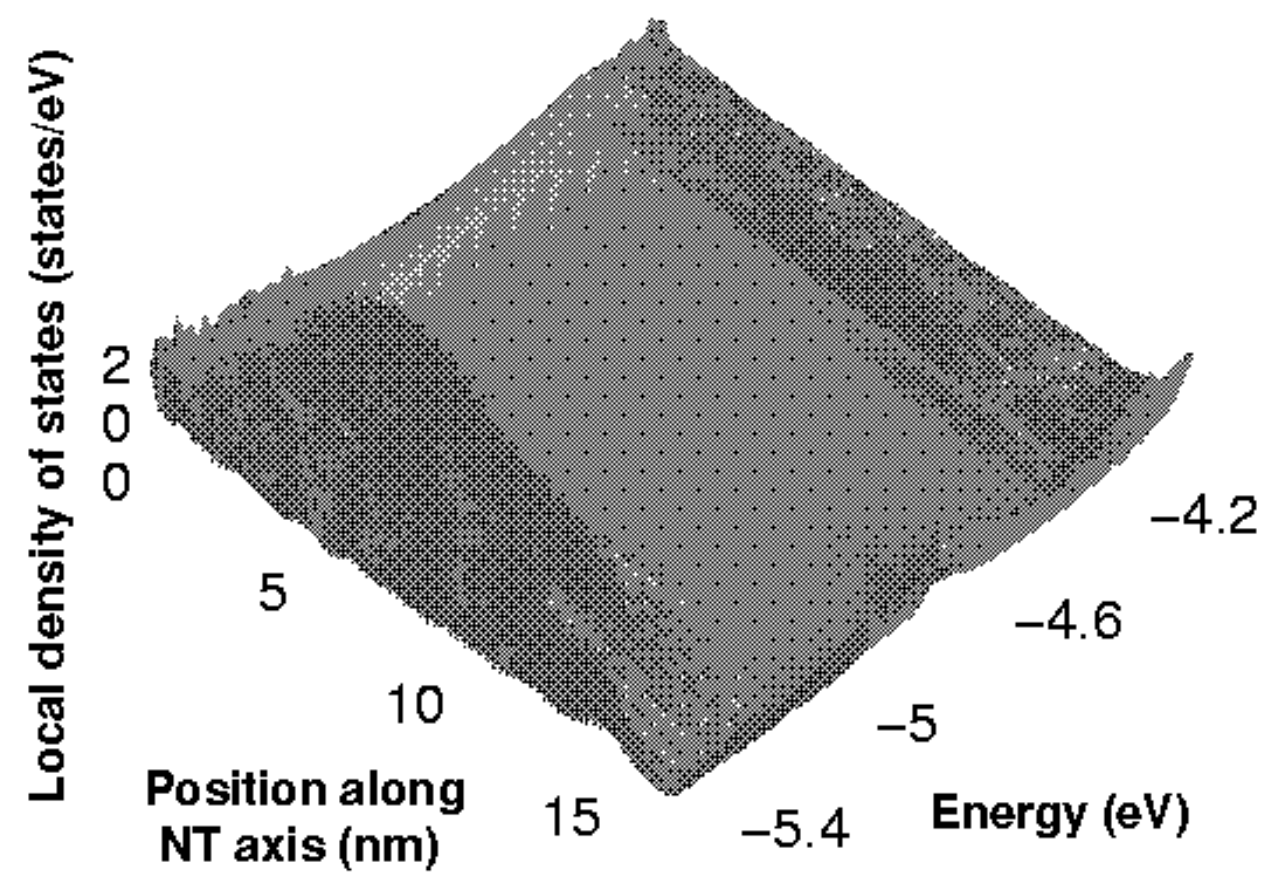

(b) L=16.9 nm: Non Self-Consistent

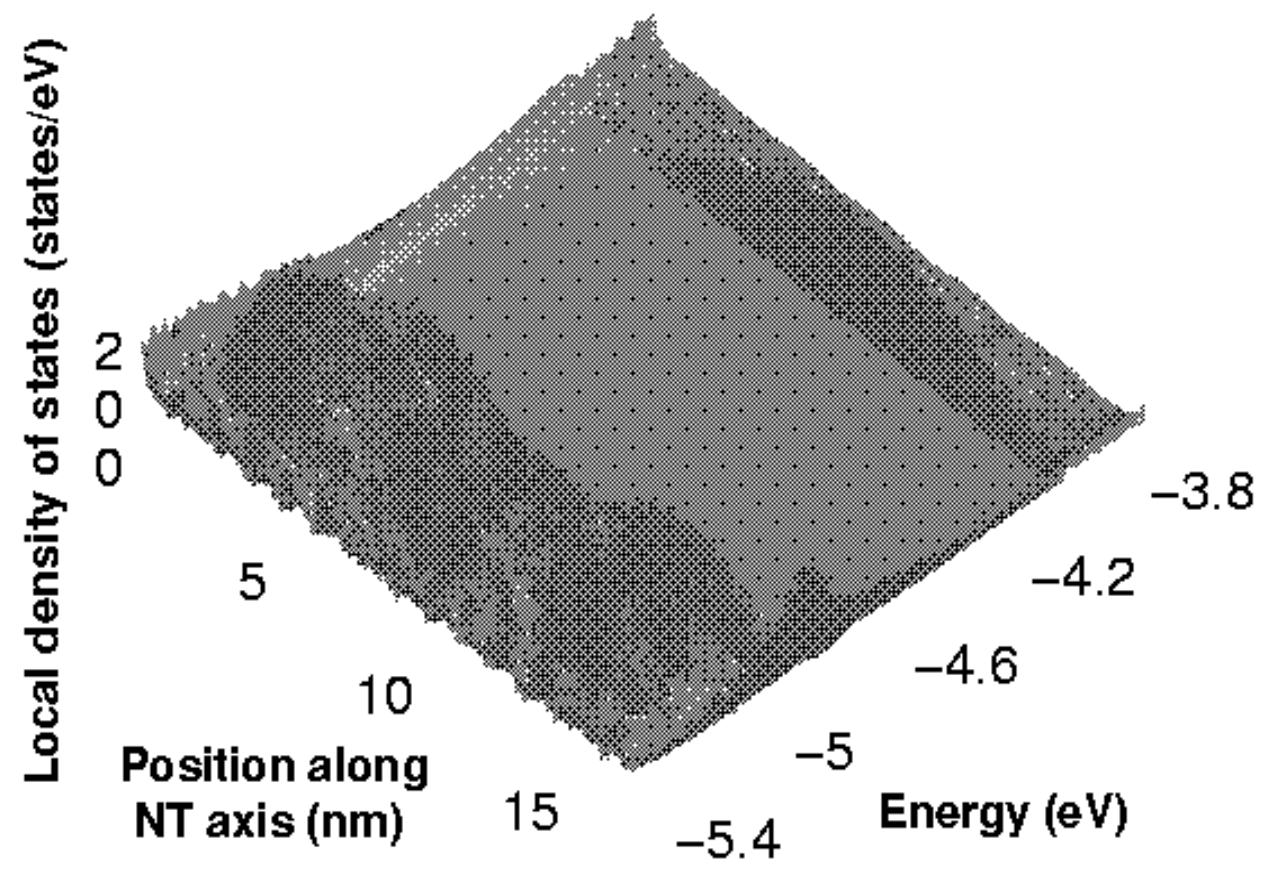

FIG. 3: (Color online) Local density of states (LDOS) as a function of position along the SWNT axis for SWNT channel length of $16.9 \mathrm{~nm}$. We show the result when self-consistent SWNT screening of the build-in electric field is included in (a). For comparison we have also shown the result for non selfconsistent calculation in (b). The plotted LDOS is obtained by summing over the 10 atoms of each carbon ring of the $(10,0)$ SWNT. Note that each cut along the energy axis at a given axial position gives the LDOS of the corresponding carbon ring and each cut along the position axis at a given energy gives the corresponding band shift. 


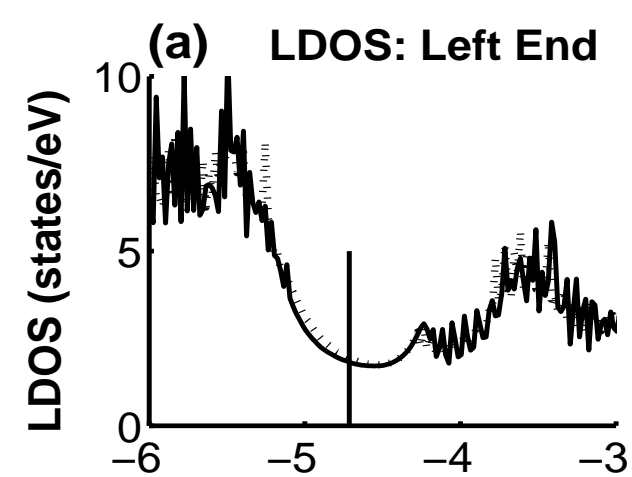

(c) LDOS: Right End

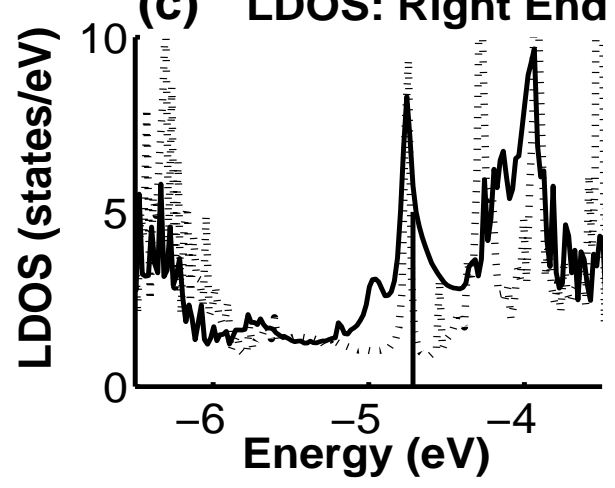

(b) LDOS: Middle

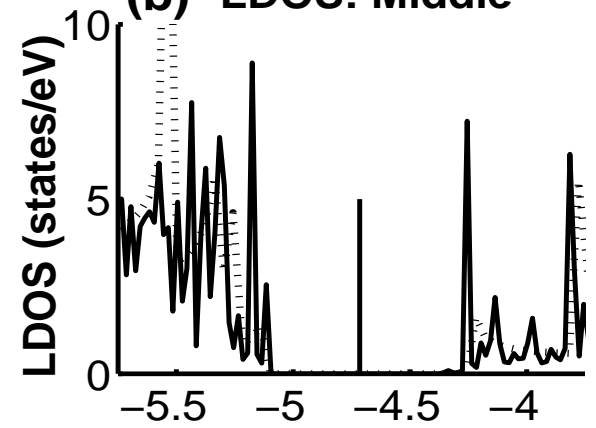

(d)

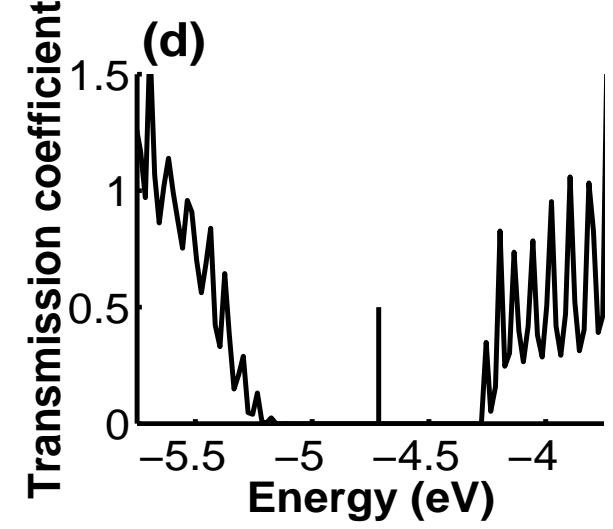

FIG. 4: Local-density-of-states (LDOS) and transmission versus energy (TE) characteristics of the 40-unit cell AuSWNT-Ti junction. (a) LDOS at the 1st unit cell adjacent to the $\mathrm{Au}$ side (left end) of the Au-SWNT-Ti junction (solid line) and the LDOS at the corresponding location of the $\mathrm{Au}-$ SWNT-Au junction (dashed line). (b) LDOS in the middle unit cell of the Au-SWNT-Ti junction (solid line) and the LDOS of the bulk $(10,0)$ SWNT (dashed line). (c) LDOS at the 1st unit cell adjacent to the Ti side (right end) of the Au-SWNT-Ti junction (solid line) and the LDOS at the corresponding location of the Ti-SWNT-Ti junction (dashed line).

(d) TE characteristics of the Au-SWNT-Ti junction. 

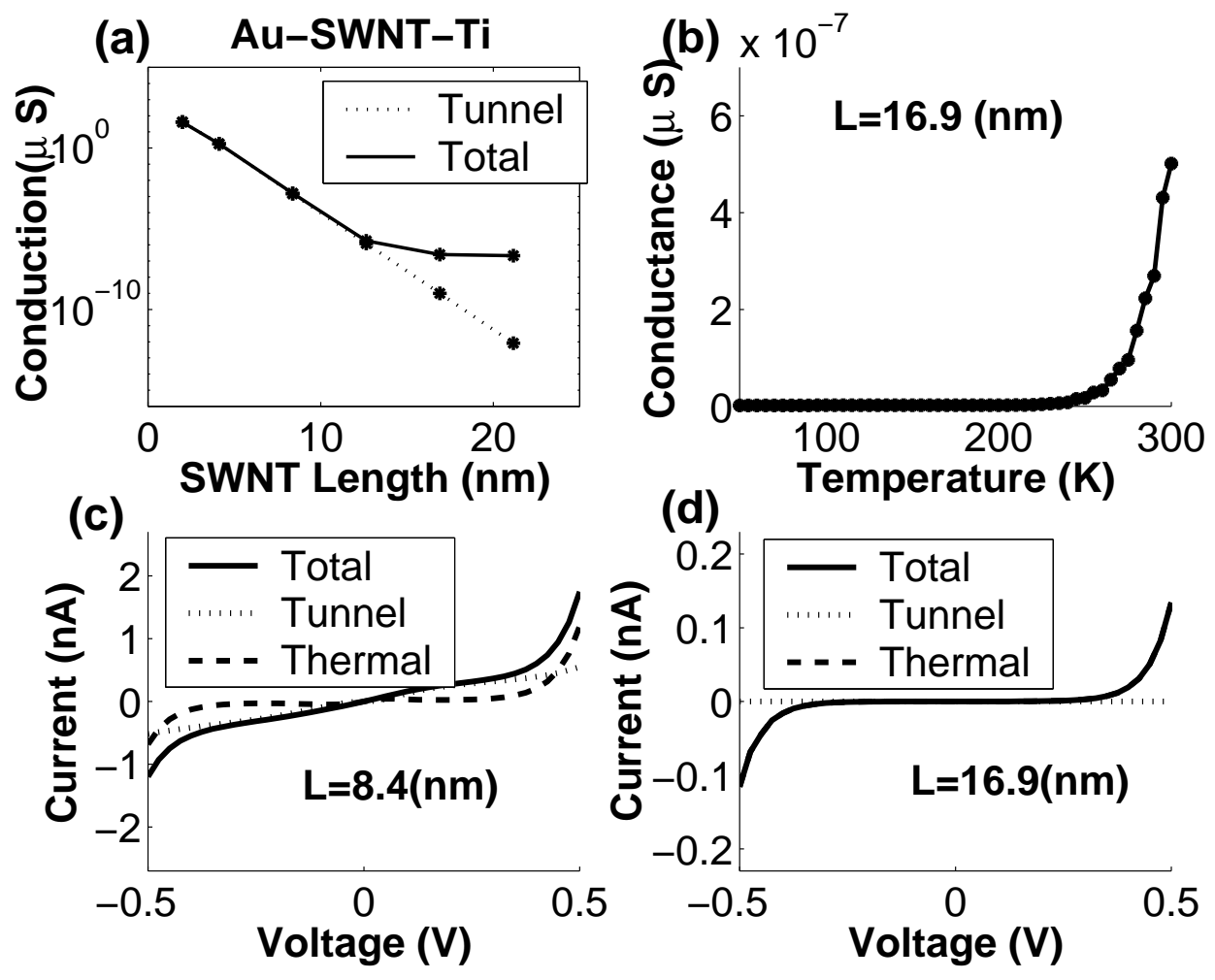

FIG. 5: (a) Room temperature conductance of the $\mathrm{Au}-$ SWNT-Ti junction as a function of SWNT channel length. (b) temperature dependence of the conductance of the 40unit cell $(16.9 \mathrm{~nm})$ SWNT junction. The room temperature current-voltage characteristics of the 20- and 40- unit cell SWNT junctions are shown in (c) and (d) respectively. 